\title{
1. Personal Transparency in Perspective: \\ The Impact of Social Networking Sites on the Recruitment Process
}

\author{
Paul Beckmann, Alexander Hoppe, Katharina Jautz, Lara Schartau, \\ Julia Schmälter
}

\section{Introduction}

It is a common myth that job seekers do not only have to take care of spelling mistakes and formal flaws in their applications anymore, but also have to 'clean up' their profiles on Social Networking Sites (SNS). In any case, this is the impression one gets when following the media. The information accessible on applicants on SNS appears, by now, to be frequently considered by Human Resource (HR) managers when they have to decide about whom to employ (Kemmer, 2009). Career centers offer workshops for job seekers on how to present themselves in social media, which is just another indicator for the fact that applicants become more transparent towards their possible future employers. Frequent media coverage of the topic and regular reports about people losing their job because of their behavior on SNS has raised the public awareness of this transparency. Our article focuses on the smallest scale of transparency, thus the accessibility of information on an individual, irrespective of what kind of information. The notion of personal transparency is a micro-perspective on the conceptual framework of transparency.

In the last decades, technological progress has served as an important impulse for growing availability and facilitating the accessibility of information in a globalized world. One catalyst for increasing transparency of individuals with regard to the significance of communication technologies is the rise of Social Networking Sites (SNS). The growing importance of SNS has changed the way people handle their information. There is a tendency towards less privacy and more disclosure of personal information and, thereby, towards greater personal transparency. Individuals, as well as companies, use Social Networking Sites to inform people about themselves and gather data about others. Although Social Networking Sites have become an integral part of everyday-life for millions of users around the world, giving a definition is appropriate as they constitute a major aspect of the analysis of this paper. Nicole Ellison depicts three main characteristics 
to define SNS. Firstly, they "allow individuals to construct a public or semi-public profile within a bounded system" (Ellison, 2007, p.211). Secondly, they provide the possibility to share the information on this profile with other individuals, and lastly they enable users to see and share the connections of other users (ibid.). For the course of this paper, the distinction between 'private' and 'professional' SNS is highly relevant. While private sites, such as Facebook, the German StudiVZ and the Dutch Hyves are used for social interaction among friends and for exchanging private information, professional SNS, such as LinkedIn and Xing are used by both, job seekers and recruiters to establish professional contacts and exchange information relevant in the professional framework of, for example, the recruitment process. Information disclosed on professional SNS can be considered to be explicitly directed to the employer and thus, merely serve as another tool for signaling job-relevant information, not giving the employer access to information which is new to him or her and, hence, no opportunity to screen the candidate. Private SNS on the other hand do not have a professional purpose but reveal personal data making the applicant's private sphere more transparent. This article examines the shifting patterns and meanings of personal transparency caused by this new dimension of social interaction.

Within the last decade, one can observe a trend towards increased personal transparency. A basic assumption of this paper is that Social Networking Sites accelerate this trend in the sense that they promote more disclosure of personal information online and, thereby, change parameters of privacy and personal transparency. Since the concept of transparency is difficult to grasp and in particular personal transparency has an inherently subjective component, it is advisable to choose an exemplary situation where personal transparency is likely to occur and is likely to have a changing impact. This paper proposes that such a scenario is found in the recruitment process and the relationships between recruiters and job seekers developed in this environment. It attempts to evaluate the job recruitment process from the point of view of transparency. By doing so, we seek to identify in how far an increased degree of information available on an individual might really have an impact, in our case on the recruitment process, a very important and decisive selection procedure that is a crucial aspect of everybody's professional life.

The two main topics discussed within the academic debate regard whether SNS are employed as a tool by recruiters within the selection process and in case they are, whether they have positive or negative consequences for both, the recruiter as well as the job seeker (e.g. Caers \& Castelyns, 2010; Kluemper \& Rosen, 2009; Clark \& Roberts, 2010). Situating our research within the academic debate on the impact of SNS on the recruitment process, our contribution stands out due to the following reasons. Firstly, while most of the existing studies focus on the USA and SNS in general, we concentrate 
on the recruitment process in Europe and private SNS in particular. As the only existent indepth analysis of the situation in Europe concentrates on Belgium, we want to add another perspective to the academic debate which is why we analyze the cases of Germany and the Netherlands. Moreover, while most studies focus on quantitative research (e.g. dimap, 2009; Jacobsmuehlen, 2010), we applied a mixed-method approach in order to add a qualitative dimension, which enables us to contribute a new and deeper insight to the current state of the academic debate. Firstly, results from a survey among recruiters and job seekers in Germany and the Netherlands will be analyzed. Secondly, a qualitative data collection was carried out through 12 interviews with Human Resource managers and marketing experts from companies with diverse backgrounds from the two countries. This approach was chosen to deliver more concise in-depth understanding of the complex interaction between the two parties involved in the recruitment and selection procedure.

This paper focuses on the question in how far the growing personal transparency through Social Networking Sites changes the relationship between recruiters and job seekers in the job selection process and claims that personal transparency has an impact on the job selection procedure. In order to tackle this research question, we first need to assess in how far SNS actually have the informational potential to initiate such a change in the job selection process. It is straightforward to argue that the selection procedure will only change due to SNS if a recruiter perceives the information on Social Networking Sites to be valuable. If the information which can be found through SNS is considered significant and trustworthy, the potential for recruiters to change the dynamics, and moreover to allow personal transparency to influence the power structure of recruitment, is likely to be exploited. As a result, if those two conditions are met, an influence on the recruitment and selection procedure is suggested. As a positive outcome of this, we consider an increased likelihood to achieve a good person-organization match, i.e. that more information that is available on an employee increases the efficiency and the quality of the candidate selection. With regard to the negative outcome, a growing likelihood of discrimination on the basis of information on SNS such as inappropriate pictures or behavior in the virtual forums might occur. Most likely, the truth will lie somewhere in between those two extremes.

In this shortened version of our original paper, we will first introduce our three leading hypotheses along which we will structure this article. Second, existing notions of transparency are outlined out of which we establish our own concept of personal transparency in the explicit case of SNS usage within the recruitment process. Moreover, we define the ideas of the changing relations of signaling and screening connected to the concept of asymmetric information and describe the influence of SNS on the varying 
relation between employers and applicants within the recruitment process. Here, we will also refer to the screening of information which might possibly influence the decision of the recruiter on whom to employ. Finally, we will deliver a short overview of the findings of our empirical research trying to examine whether our leading hypotheses can be confirmed. A concluding section will summarize our findings and seek to offer an outlook into the future, pointing at possible consequences resulting from the use of Social Networking Sites within the selection process and the potential change in power relations between recruiters and applicants.

\section{Conceptual Framework}

In order to evaluate the empirical data we collected, it is necessary to establish the theoretical framework into which we embed our analysis. Hence, we develop and explain the concepts which we will apply throughout our paper in the following section. Transparency as such is a concept that is broadly discussed and frequently conceptualized in academic literature. Starting out from its definitions in political and economic context, we will develop our understanding by focusing on the individual. Subsequently, we discuss the relation between recruiter and applicant introducing some of the concepts which are at the core of our analysis: asymmetric information, signaling and screening. On the basis of the data gained with these new possibilities, the chance of better matches between applicant and organization as well as the discrimination of certain groups of people might become more apparent.

Assuming that the disclosure of personal information online renders an individual more transparent than before, we hold that there are significant implications of this development. Since the process of increasing transparency is difficult to grasp in its entirety, our research assesses one particular situation where this development is likely to surface considerably, namely the job recruitment process. As the relationship between recruiter and job seeker is sensitive and heavily depends on exchange of information, an assumed increased availability of information through personal transparency is likely to have a noticeable impact on changing the power structure in this relationship. In order to tackle this question, we have developed three leading hypotheses that guide the research and the analysis of this paper.

LH 1) Increased personal transparency changes the relationship between recruiter and job seeker in the selection process, i.e. adds another screening option to recruiters' possibilities of gaining information. 
LH 2) Information from SNS is valuable in order to assess an individual's personality for the purpose of the selection process.

LH 3) Increased personal transparency has an impact on applicant selection on the labor market.

To understand the findings of our empirical research in their theoretical context, we need to discuss the concept of transparency and, in particular, personal transparency. The following section will debate definitions of transparency adequate for the framework of this paper and further develop a new definition grasping the implications of personal transparency. This newly designed concept will focus on transparency of the individual within Social media and thereby combine elements of existing definitions with the particular conditions inherent to the situation of recruitment processes.

One important conceptualization is provided by David Heald (2006), who identifies two distinct dimensions applicable to our conceptualization which cover different directions of transparency: upwards and downwards (pp. 27). These require a hierarchical relationship between the actors involved. According to Heald, transparency upwards means "that the superior/principal can observe the conduct, behavior, and/or results of the subordinate/agent" (p. 27). On the contrary, transparency downwards is directed the other way around, so that the subordinate is able to observe his or her superior. We refer to the job recruitment process as a hierarchical situation as the recruiter holds the power of decision whom to employ. This means that the recruiter is able to access information on the job seeker (transparency upwards) by monitoring what takes place within his or her SNS profile, whereas this is not possible vice versa (transparency downwards).

However, as these notions of transparency are not sufficient to cover all levels of transparency important for our analysis, we also apply G. Randolph Mayes' (n.d.) classification into uni- and omnidirectional transparency. Here, unidirectional transparency occurs when only one of Heald's dimensions is fulfilled. This means that information of one actor is accessible to another actor, while the latter is able to secure his or her own information from intrusion from outside. In contrast, omnidirectional transparency concerns a "society in which the activities of private citizens, multinational corporations, and government are all becoming easier for anyone to observe" (p. 18) which means that in theory, everybody has access to information about all actors towards which he or she is also transparent. The following section establishes the correlation between these conceptualizations, and introduces our own perception of personal transparency within SNS and the job recruitment process. 
First, this article focuses on omnidirectional transparency which can be applied within an online community as each individual discloses the same level of information he is able to access himself. Moreover, the relation between members and people outside of the SNS community can be considered significant. Second, in this case, the information disclosed by members is retrieved by non-members, although the members within the community do not have access to the non-member's private spheres nor can they identify the people viewing their profiles, hence this relationship represents unidirectional transparency. The third step in developing our concept scrutinizes the concrete example of the relation between job recruiter and job seeker. We consider this relationship to demonstrate transparency upwards, as the superior, the recruiter, can access information about the individual job seeker, whereas the job seeker cannot control whether his information is being checked.

By correlating our previously discussed notions, we introduce the concept of personal transparency which we apply in our analysis (Graphic 1). Here, the individuals within the Social Networking Site community are interrelated in an omnidirectional transparent situation. On the contrary, between members and non-members of the community transparency can be considered unidirectional, since SNS members do not have the opportunity to access data from non-members. Moreover, we consider the relation between job recruiter and job seeker as one of transparency upwards, as the recruiter can be considered as hierarchically superior to the applicant in the job recruitment process.

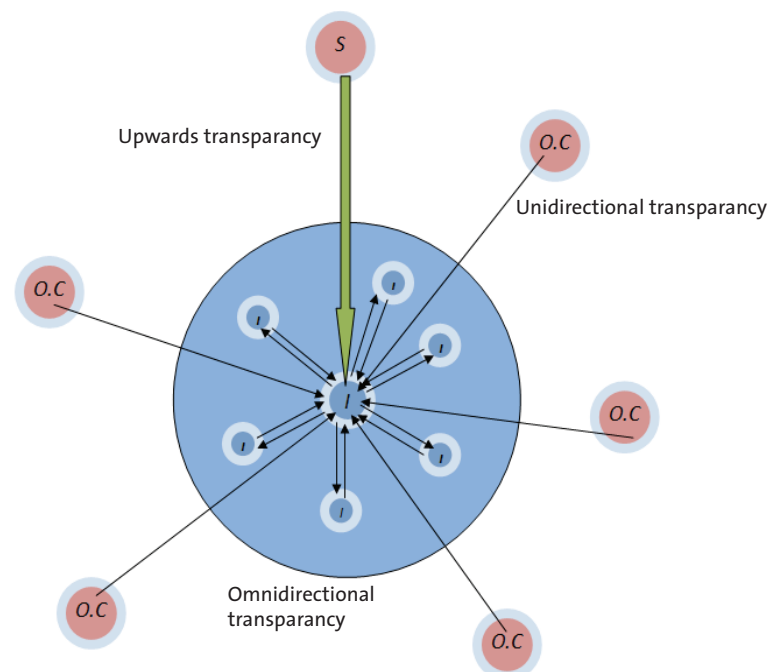

Graphic 1 


\section{Asymmetric Information}

In the following section, we will concentrate on the theories of information asymmetry, signaling and screening, which will firstly be defined and then applied to the recruitment process. We will clarify in how far we assume the growing transparency conceptualized above to change the relationship between job seeker and recruiter and to adjust the situation of asymmetric information.

The concept of asymmetric information describes a situation where two parties involved in a transaction have different levels of information about the value of the exchanged good. The concept is derived from economics literature and has firstly been put forward by Akerlof (1970). It refers to a particular distribution of information and the implication of this unequal distribution for the assessment of a situation. In his seminal work about this concept, Akerlof evaluates the consequence of such an unequal distribution of information, concluding that it leads to a situation of adverse selection. Spence (1973) applied the case of asymmetric information to the labor market. He argued that an employer had significantly less information about the productivity and capability of a job candidate, compared to the knowledge of the applicant. As a result, the adverse selection would also lead to a crowding out of highly capable workers on the labor market if high abilities would cease to be rewarded. Consequently, it has been argued that highly productive and highly capable workers would invest in education to signal their abilities with the eventual goal of receiving a return on the investment into education. On this basis, employers could then reasonably select job applicants and receive a more productive worker for their firm.

A further contribution to the discussion has been published by Stiglitz (1975). It deals with the same questions of imperfect information but then asks the question of what the buyer could do to improve his market outcome. The basic idea is that the buyer should screen the product that he wants to receive in order to improve his information about it. The original example deals with insurance. Since an insurance company has less information about the risk of an insured person, it has to try to improve his knowledge in order to avoid the adverse selection which would be a crowding out of low-risk persons by people with higher risks. With regard to the job selection process, screening refers to the ability of an employer to retrieve information about an applicant.

It is straightforward to assume that signaling and screening interact. The more an applicant signals to an employer, the more the employer can eventually then screen about an applicant and vice versa. However, in the application of the labor market, it is more likely that the information exchange traditionally starts with the signaling model since it is more likely that the candidate first sends information to the employer, i.e. the 
uninformed party then responds to the information exchange initiated by the informed party. Contextualizing this into our overall research framework of personal transparency, we assume that the easy accessibility of information on SNS changes precisely this mechanism, as the role of the recruiter is strengthened.

\section{Discussion}

In the following section we will discuss our findings along the lines of our three leading hypotheses.

$\left.L H_{1}\right)$ Increased personal transparency changes the relationship between recruiter and job seeker in the selection process, i.e. adds another screening option to recruiters' possibilities of gaining information.

Regarding our first leading hypothesis, we found that every second recruiter uses internet research and SNS to gather additional information on their applicants and regards it legitimate to do so, which influences the frequency of using SNS. This reveals a clear potential for a change in the relationship between employers and job seekers both in Germany as well as in the Netherlands as the recruiter gains a further tool to enhance his or her superior position within the selection process. Furthermore, as younger recruiters tend to make more use of SNS, the next generation is even more likely to experience a changed relationship. Job seekers seem to be aware of this changing relation and appear concerned about their privacy, and, thus, do not consider the use of SNS in the recruitment process legitimate. This leads to an increased number of job seekers restricting the access to their profile.

Having seen that recruiters might alter their selection behavior due to the existence of additional information, it becomes apparent that there is a large potential for changing dynamics in the job recruitment through increased personal transparency driven by Social Networking Sites and with it, greater informational discretion for employers. Accordingly, we hold that the basic condition for an implication of increased personal transparency is given. However, it is ambiguous if this source of information might really be exploited. This depends on the quality of the information that can be derived from Social Networking Sites, or at least the perception thereof. The dynamics in the relationship between recruiters and job seekers are only likely to change if recruiters would consider the insight from Social Networking Sites as worthwhile and trustworthy. Only if they did so, are employers likely 
to attach less importance to the applicants' signals, thereby ascribing more attention to additional information retrieved from Social Networking Sites. Accordingly, our second leading hypothesis assumed that

$\mathrm{LH}$ 2) information from SNS is valuable in order to assess an individual's personality for the purpose of the selection process.

Our research found that neither recruiters nor job seekers perceive information disclosed on SNS to be indicative of true character traits. Thus, the frequent use of SNS within the selection process in order to choose a future employee seems concerning, as the value of the information is questionable but they still offer a possible ground for discrimination. Hence, the validity of the second leading hypothesis has to be neglected. According to the perceptions of both recruiters and job seekers, many important character traits are not evaluable through Social Networking Sites. Moreover, the most valid information for employers is usually restricted, and the information disclosed appears to be hardly reliable. Obviously, this low informational value also has a negative impact on the potential of SNS for influencing the relationship of recruiter and job seeker within the job recruitment process.

The last hypotheses that

LH3) Increased personal transparency has an impact on applicant selection on the labor market

requires a complex answer. The inclusion of Social Networking Sites by recruiters for the purpose of gathering additional information about the applicants might have several consequences, both of positive and negative nature. A possible positive consequence might be the increase in potential chances to find an employee who matches the corporate culture of the company. This might result from the recruiters' possibility to gain further data about the applicant on SNS. However, a vast majority of recruiters denied that Social Networking Sites would improve the chance to find a better person-organization match which is a logical consequence of the recruiters' general perception that the value of information disclosed on SNS is rather low for the purpose of assessing an applicant's personality. In line with that, job seekers indicate disbelief in the chances of improving the correspondence between employee and company as well. 
On the other hand, negative consequences might result from the inclusion of SNS within the hiring process, namely if recruiters use the information gained in order to discriminate particular groups of applicants. According to our findings, there is a general belief of both parties that discrimination is apparent in job recruitment and can be enhanced by the inclusion of information revealed on SNS. Hence, we can conclude that the changing relationship within the hiring procedure due to the usage of SNS does arguably not lead to positive consequences as an improved person-organization match, but does rather have negative influences on the job selection process. This might result from the fact that the value of information disclosed on SNS profiles is generally conceived as not trustworthy and therefore, does not offer a basis for recruiters to correctly perceive the personality of job seekers. Thus, the changing power relations between recruiters and job seekers arguably carries merely disadvantages for the applicants within the job recruitment process.

To sum up, SNS seem insufficient for leading to a better person-organization match since character traits cannot be identified by screening Social Networking Sites which is a precondition for assessing the match between a company's values and individual preferences. Discrimination, however, cannot be ruled out easily since a negative first impression is more likely to arise from SNS than a positive one. Thus, if there is an influence on the interaction between recruiters and job seekers through the inclusion of Social Networking Sites, this impact is more likely to lead to a disadvantage for the applicants.

\section{Implications for personal transparency}

Our case study revealed several insights which can be applied to our overall research framework of personal transparency. In order to answer our research question, namely in how far the growing personal transparency through Social Networking Sites changes the relationship between recruiters and job seekers in the job selection process, we now turn to discuss several broader implications that have only been touched upon so far.

Firstly, it is reasonable to suggest that the more people consider the inclusion of SNS in the recruitment process legitimate, the more likely is the growth of the use of SNS in the future. Our empirical evidence shows that there are important discrepancies in the perception of legitimacy. The perception of the recruiters in that case is more influential, since they are the ones who decide on the usage, unless a stronger public discourse calling for action in that area arises. Obviously, people are willing to disclose information online in a horizontal way, i.e. for people who use SNS as a tool for private interaction and communication (omnidirectional transparency). However, if this situation is turned into a vertical one, i.e. when outsiders who can be considered hierarchically superior make use of the transparency of the network members (unidirectional transparency) in order 
to improve their bargaining position, people consider this as an illegitimate intrusion into their private sphere. Whether people consider the use of SNS as legitimate or not, strongly depends on their attitude towards privacy in general. According to our results, the preference for privacy scores very high and similarly in Germany and the Netherlands, whereas Germans consider the use of SNS as less legitimate than Dutch, in particular among employers.

Secondly, the perception of screening applicants through SNS in Germany even led to the legislative proposal prohibiting the application of information gathered from Social Networking Sites for recruitment purposes (Deutsche Bundesregierung, 2010). It is questionable in how far such a legislative regulation will lead to the desired goal, just as every anti-discrimination law faces problems in enforcement. However, the mere existence of legislation is likely to raise the moral threshold of utilizing this source of information. Our interviews show that Germans preferred to ascribe more attention to increasing the awareness about personal transparency online and favor more efforts towards media pedagogy passed on by parents, schools, and the state. The fact, however, that the issue at hand has entered the national parliamentary debate in Germany shows that it is a highly salient topic. Yet the issue is obviously observed differently in the Netherlands. Probably rooted in a generally more liberal societal and political culture, the Dutch seem to assign more responsibility to the individual and are thus not in favor of governmental regulation. We do not intend to make any ethical and generalizing judgments about the righteousness of growing personal transparency in society. Nevertheless, this article suggests increasing the public discourse on this issue and highlighting several key effects of this development.

Thirdly, relating this to our research question, we come to the result that there is the potential for an impact on the recruitment process and our evidence shows that if there are effects on the recruitment process derived from growing personal transparency, they are more likely to be negative. As a result, personal transparency poses several risks to the users of Social Networking Sites. Although there is already a general awareness about privacy issues in Social media, there are only two ways to deal with increased personal transparency and its potential to be exploited by outsiders of the community. On the one hand, one could change his behavior on Social Networking Sites in order to foreclose any detrimental effect for oneself. On the other hand, the use of SNS for purposes other than the original social interaction could be regulated in whatsoever form, be it legally, as already attempted in Germany, technically or through any other measure. We acknowledge, however, that SNS do not only have a negative potential for the recruitment process, but also a positive one. Using social media to present oneself to future employers 
adds another option for job seekers to find an employment. We argue that professional SNS such as LinkedIn are perfectly suitable to perform these tasks and also provide a clear demarcation of private and public sphere, something that is blurred within private SNS. Such a division between professional SNS as a venue of self-promotion and 'head hunting' and private SNS for social communication only would be recommendable, also to re-build feelings of security and confidence towards virtual communication and the virtual world as a whole.

In general, the rise of the internet has moved the perceived borders of privacy, and thus opened up possibilities to screen personal data. Hence, we can transfer our conclusions to broader societal issues. Not only the recruitment process, but also other fields such as personalized advertising and, in general, personal interactions have the potential to change through increased personal transparency. It is appropriate to transfer our two possible remedies (individual responsibility or regulation) to other cases dealing with personal transparency beyond the recruitment process. Concerning the first remedy, namely individual responsibility, we strongly advise to further increase the awareness and sensitivity about the disclosure of personal information. In our opinion, how to deal with personal data should be made an integral part of general education.

This is supported by evidence gained through our qualitative research. As a second remedy, we propose to regulate the use of SNS in certain particularly sensitive issues in a way that the information from Social media cannot be exploited by community outsiders or insiders for inappropriate purposes any longer. However, the latter solution poses some challenges related to freedom of expression as only a fine line separates regulation from censorship within the open medium of the internet. Openness of information is an intrusive element of Social Networking Sites so that too much restriction might fundamentally change their character and diminish their popularity. 


\section{Conclusion}

In our paper, we have investigated possible influences of growing personal transparency through Social Networking Sites. For the purpose of giving a better insight into this process, we carried out a case study examining the influence of growing personal transparency on the recruitment process. We introduced the underlying basic theoretical concepts before presenting the results of our quantitative and qualitative empirical studies. Subsequently, those findings have been contextualized and the validity of our main hypotheses has been assessed.

Concerning our empirical study, we have to acknowledge several shortcomings. Firstly, since it is a sensitive issue, we cannot guarantee that our results are free of any bias, as people might be reluctant to answer honestly. Secondly, our samples have not been equally distributed among the different groups that we were inquiring. Thirdly, the respondents to the job seekers questionnaire have mostly been invited through Social Networking Sites, thus we might suffer from a pro-SNS bias in those responses. Moreover, we cannot be entirely sure that only HR managers have filled in the recruiters' questionnaire since we only sent it to companies as such. However, we expect that the majority of those invitations have been forwarded to HR departments and can thus be treated as valid.

Our empirical data delivered several revealing insights into the process of recruitment on the job market and showed that growing personal transparency through Social Networking Sites clearly has the potential to change the relationship between recruiters and job seekers. We found that additional information available has the potential to distract recruiters from the originally transmitted information presented on a traditional application. Although the information accessible through Social Networking Sites is perceived rather inappropriate for correctly assessing a job candidate, there is possibly an impact on the candidate selection triggered by SNS since employers make use of it. This influence is more likely to result in negative than in positive effects for the job seeker. For the purpose of overcoming this problem, we suggest two possible solutions. Firstly, the general awareness about possible implications of personal transparency needs to be raised. Our paper contributes to increasing this awareness by highlighting potential consequences on the recruitment process. Secondly, a stricter regulation on the use of Social Networking Sites with the aim of restricting non-private use might help to foreclose possible detrimental outcomes for individuals.

The observed insights can be used to generalize on personal transparency as a whole. It appears that the development towards increased transparency of the individual is likely to continue. SNS are one major driving force of this growth. However, there are multiple 
other contributing factors, such as public surveillance and personalized advertisement. This is in line with a general trend towards more transparency in contemporary society, such as institutional transparency in modern governments. Yet this trend will probably provoke a growing opposition and possible counter-reactions. As it has been put by Hood (2007) "there is no doctrine [...] without its counter-doctrines, and [...] transparency is not an exception to that rule" (p.20). Concerning the recruitment process, we might already be able to observe the counter-movement in action. German policy-makers addressing this question, repeated media coverage on SNS privacy issues, and campaigns for responsible dealing with Social Networking Sites reflect this suggestion.

It is going to be very interesting to observe how these societal processes will continue. Moreover, it will be of particular interest to look across national borders since societal and political cultures and norms presuppose different ways to deal with the issue. Our paper only touched upon possible different reactions on and attitudes towards personal transparency in Germany and the Netherlands. Drawing on cultural studies, this transnational perspective might be an interesting starting point for further research. Moreover, with regard to the recruitment process, a research framework similar to ours might want to take professional Social Networking Sites such as LinkedIn into account, for example, in order to further elaborate on the division between private and public issues in the virtual world. Additionally, the impact of growing personal transparency on other sectors of everyday life such as interpersonal relationships might be worth investigating. Our paper can serve as the basis for further research of this kind, as it provides a comprehensive analysis of the impacts personal transparency can have on society, founded on empirical evidence from a detailed case study. We did not intend to make any ethical and generalizing judgments about the righteousness of growing personal transparency in society. Nevertheless, this article suggests to increase the public discourse on this issue and to highlight several key effects of this development to which we hope to contribute with this analysis. 


\section{Bibliography}

Akerlof, G. A. (1970). The market for “lemons”: Quality uncertainty and the market mechanism. The quarterly journal of economics, 84(3), 488-500.

Caers, R. \& Castelyns, V. (2010). LinkedIn and Facebook in Belgium: The Influences and Biases of Social Network Sites in Recruitment and Selection Procedures. Social Science Computer Review, 1-12.

Clark, L.A. \& Roberts, S.J. (2010). Employer's Use of Social Networking Sites: A Socially Irresponsible Practice. Journal of Business Ethics, 95, 507-525.

Deutsche Bundesregierung. (2010). Entwurf eines Gesetzes zur Regelung des Beschäftigtendatenschutzes. (17/4230). Berlin: Deutsche Bundesregierung dimap. (2009). Umfrage zu Haltung und Ausmaß der Internetnutzung von Unternehmen zur Vorauswahl bei Personalentscheidungen. Retrieved June $6^{\text {th }}$, 2010, from: http://www.bmelv.de/SharedDocs/ Downloads/Verbraucherschutz/InternetnutzungVorauswahIPersonalentscheidungen. pdf?_blob=publicationFil

Ellison, N.B. (2007). Social Network Sites: Definition, History and Scholarship. Journal of Computer-Mediated Communication, 13/1 210-230.

Heald, D. (2007). Varieties of Transparency. In C. Hood \& D. Heald (Eds.). Transparency: The Key to Better Governance? Oxford/ New York: Oxford University Press.

Hood, C. (2007). Transparency in Historical Perspective. In C. Hood \& D. Heald (Eds.). Transparency: The Key to Better Governance? Oxford/ New York: Oxford University Press.

Jacobsmühlen, T. (2010). Social Media Report HR 2010. Retrieved June $6^{\text {th }}, 2010$, from: http://www.wirtschaftsblatt.at/images/uploads/o/6/d/409709/social_media_report_ hr_201020100224151102.pdf

Kemmer, C. (2009). Private Internet-Daten als Problem bei der Bewerbung. Retrieved June $15^{\text {th }}, 2011$, from http://www.welt.de/News/article4370702/Private-Internet-Daten-alsProblem-bei-der-Bewerbung.html

Kluemper, D.H. \& Rosen, P.A. (2009). Future employment selection methods: evaluating social networking web sites. Journal of Managerial Psychology, 24(6), 567-580. Mayes, G.R. (n.d.). Privacy in a Transparent Society. retrieved May, $15^{\text {th }} 2011$ from http://www.csus.edu/ indiv/m/mayesgr/Scribble/Privacy\%20Stuff/Privacy\%20in\%20a\%20Transparent\%20 Society.pdf

Spence, M. (1973). Job market signaling. The quarterly journal of Economics, 87(3), 355. 